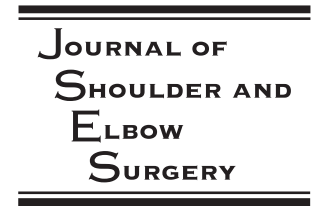

www.elsevier.com/locate/ymse

\title{
Importance of a three-dimensional measure of humeral head subluxation in osteoarthritic shoulders
}

\author{
Alexandre Terrier, $\mathrm{PhD}^{\mathrm{a}, *}$, Julien Ston, $\mathrm{MSc}^{\mathrm{a}}$, Alain Farron, $\mathrm{MD}^{\mathrm{b}}$
}

\author{
${ }^{a}$ Laboratory of Biomechanical Orthopedics, Ecole Polytechnique Fédérale de Lausanne, Lausanne, Switzerland \\ ${ }^{b}$ Service of Orthopaedics and Traumatology, University Hospital Center and University of Lausanne, Lausanne, Switzerland
}

\begin{abstract}
Hypothesis: During total shoulder arthroplasty (TSA), humeral head subluxation may be difficult to manage. Furthermore, there is a risk for postoperative recurrence of subluxation, affecting the outcome of TSA. An accurate evaluation of the subluxation is necessary to evaluate this risk. Currently, subluxation is measured in 2 dimensions (2D), usually relative to the glenoid face. The goal of this study was to extend this measure to 3 dimensions (3D) to compare glenohumeral and scapulohumeral subluxation and to evaluate the association of subluxation with the glenoid version.

Materials and methods: The study analyzed 112 computed tomography scans of osteoarthritic shoulders. We extended the usual 2D definition of glenohumeral subluxation, scapulohumeral subluxation, and glenoid version by measuring their orientation in $3 \mathrm{D}$ relative to the scapular plane and the scapular axis. We evaluated statistical associations between subluxation and version in $2 \mathrm{D}$ and 3D.

Results: Orientation of subluxation and version covered all sectors of the glenoid surface. Scapulohumeral subluxation and glenoid version were highly correlated in amplitude $\left(R^{2}=0.71 ; P<.01\right)$ and in orientation $\left(R^{2}=0.86 ; P<.01\right)$. Approximately every degree of glenoid version induced $1 \%$ of scapulohumeral subluxation in the same orientation of the version. Conversely, glenohumeral subluxation was not correlated to glenoid version in $2 \mathrm{D}$ or in $3 \mathrm{D}$.

Conclusions: Orientation of the humeral subluxation is rarely within the arbitrary computed tomography plane and should therefore be measured in 3D to detect out-of-plane subluxation. Scapulohumeral subluxation and glenoid version measured in 3D could bring valuable information for decision making during TSA.

Level of evidence: Basic Science, Anatomy, Imaging.

(c) 2015 Journal of Shoulder and Elbow Surgery Board of Trustees.
\end{abstract}

Keywords: Humeral head subluxation; glenoid version; total shoulder arthroplasty; CT scan; 3D reconstruction

*Reprint requests: Alexandre Terrier, $\mathrm{PhD}$, Laboratory of Biomechanical Orthopedics, Ecole Polytechnique Fédérale de Lausanne, Station 19, CH-1015 Lausanne, Switzerland.

E-mail address: alexandre.terrier@epfl.ch (A. Terrier).
Although total shoulder arthroplasty (TSA) is a successful technique to treat primary glenohumeral osteoarthritis, complications have been specifically associated with cases in which shoulders have high preoperative humeral head subluxation. ${ }^{9,15}$ It is usually assumed that posterior subluxation of the humeral head can lead to posterior 
erosion of the glenoid. ${ }^{10}$ Furthermore, there is a risk of recurrence of the subluxation after TSA that may be responsible for wear and early loosening of the glenoid component. $^{2,4,6}$ Although a causality link between humeral head subluxation and glenoid version of osteoarthritic shoulders is intuitively expected, no statistical correlation has been reported yet. ${ }^{1,4,7,8,10,16,17}$

The most accepted measurement of humeral head subluxation was derived by Papilion and Shall. ${ }^{12}$ It was then adapted by Badet and Walch ${ }^{1,17}$ and used by Walch to propose its classification of glenohumeral osteoarthritis. ${ }^{16}$ They defined the glenohumeral subluxation as the percentage of humeral head offset from the glenoid axis relative to the humeral head diameter. This measure is calculated in 2 dimensions (2D) in a computed tomography (CT) slice approximately in the middle of the glenoid surface. Since Badet and Walch, other techniques to measure the subluxation have been developed.

However, the glenoid surface might not be the optimal reference to evaluate the subluxation. The humeral head can indeed perfectly face the glenoid fossa but be highly unaligned with the scapula and the muscle action lines. This is particularly true when the glenoid is significantly eroded or dysplastic. Accordingly, Walch recently proposed using the scapula as a reference to measure the subluxation by evaluating the eccentricity of the humeral head center from the Friedman (scapular) axis. 10,11,13

The glenohumeral subluxation is measured in the arbitrary plane of CT images, but subluxation occurs in all directions. ${ }^{5}$ To date, there is no 3-dimensional (3D) method to measure the humeral head subluxation out of the CT plane, as there is for the glenoid version. Therefore, the goal of this study was to evaluate the advantage of a 3D measurement of shoulder subluxation and to test its correlation with the 3D measure of glenoid version. Thus we compared the glenohumeral and scapulohumeral subluxations in $2 \mathrm{D}$ and $3 \mathrm{D}$.

\section{Materials and methods}

We performed a retrospective case-control study of 2D and 3D measurements of subluxation and version on a consecutive series of osteoarthritic shoulders for regular TSA planning. We included 78 female and 34 male patients with an average age of 71 years (range, $44-89$ years).

\section{Shoulder subluxation}

Glenohumeral and scapulohumeral subluxations were first measured in 2D according to the standard method proposed by Walch $^{16}$ and extended by Kidder. ${ }^{10}$ The $2 \mathrm{D}$ subluxations were adapted such that a centered head had $0 \%$ subluxation instead of $50 \%$. A subluxation of $55 \%$ according to Walch was reported as a $5 \%$ posterior subluxation in this study.

We extended the 2D glenohumeral and scapulohumeral subluxations to $3 \mathrm{D}$ to evaluate not only the amplitude but also the orientation of the subluxation. We defined the 3D glenohumeral subluxation as the relative distance between the humeral head center and the glenoid center projected onto a plane perpendicular to the glenoid centerline (Fig. 1). We defined the 3D scapulohumeral subluxation with the same distance but projected onto a plane perpendicular to the scapular axis. The 2 projected distances were normalized to the humeral head diameter. A subluxation of $0 \%$ corresponded to a centered head, whereas $50 \%$ corresponded to a subluxation distance equivalent to the humeral head radius. The orientation of the 3D subluxation was the polar angle of the humeral head center relative to the glenoid center and the anteriorposterior axis (Fig. 1). The orientation of the glenohumeral subluxation was measured in a plane perpendicular to the glenoid centerline, whereas the orientation of the scapulohumeral subluxation was measured in a plane perpendicular to the scapular axis.

\section{Glenoid version}

The 2D glenoid version was measured according to a method proposed by Friedman ${ }^{3}$ and used by Walch. ${ }^{16}$ This $2 \mathrm{D}$ version was adapted so that it was always positive. A version of $-10^{\circ}$ was reported as $10^{\circ}$ posterior in this study.

We extended the $2 \mathrm{D}$ glenoid version measure to $3 \mathrm{D}$ so that we could obtain its orientation and compare it to the $3 \mathrm{D}$ subluxation measures. ${ }^{14}$ The $3 \mathrm{D}$ version was the angle between the glenoid centerline and the scapular axis (Fig. 1). The orientation of the version was the angle between the glenoid centerline and the anterior-posterior axis. The orientation of the version was measured in a plane perpendicular to the scapular axis.

\section{D analysis and bone landmarks}

The 3D definitions of glenohumeral subluxation, scapulohumeral subluxation, and glenoid version required 5 anatomic quantities: the scapular plane, the scapular axis, the glenoid centerline, the glenoid center, and the humeral head center. These anatomic quantities were defined from bone landmarks that were placed on a 3D reconstruction of the scapula and the humerus. ${ }^{14}$ The scapular plane was fitted on 5 points along the supraspinatus fossa and on 5 points along the axillary border. The scapular axis was fitted on the same 5 points of the supraspinatus fossa, projected onto the scapular plane. The anterior-posterior axis was perpendicular to the scapular axis and the scapular plane. The glenoid surface was identified on the $3 \mathrm{D}$ reconstruction. We defined the glenoid center as the centroid (geometric center) of the glenoid surface projected onto the glenoid surface. The glenoid centerline was the axis passing through the glenoid center and the center of a sphere fitted on the glenoid surface. The humeral head center was the center of a sphere fitted on 5 landmarks placed manually. One point was placed at the infraspinatus insertion center, 1 point at the upper part of the bicipital groove, and 3 points on the articular surface (superior, middle, inferior). CT segmentation and landmark positioning were achieved with the visualization software Amira (Visage Imaging $\mathrm{GmbH}$, Berlin, Germany). The geometric analysis was done with MATLAB (MathWorks, Inc, Natick, MA, USA).

\section{Statistical analysis}

In 3D, we evaluated the distribution of orientation of glenohumeral subluxation, scapulohumeral subluxation, and glenoid version 

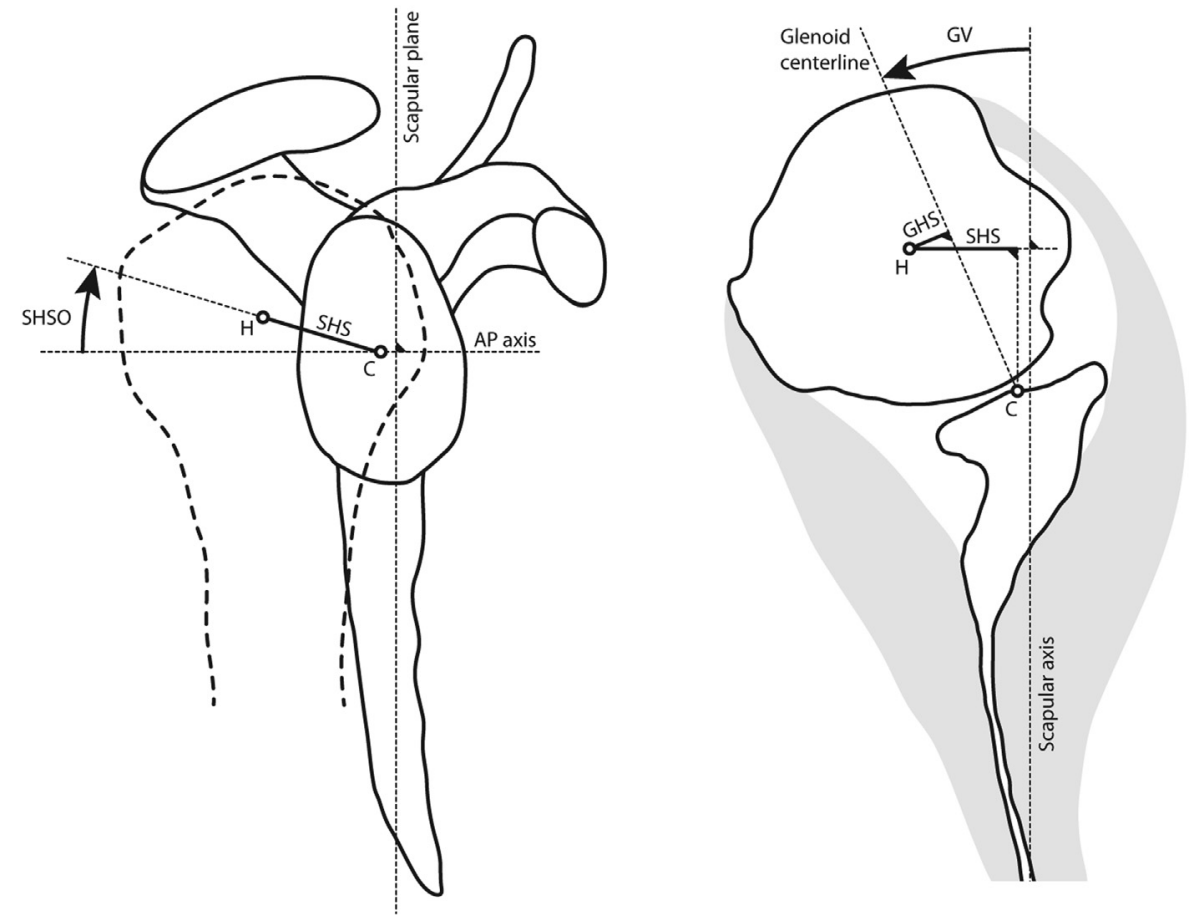

Figure 1 The 3D scapulohumeral subluxation $(S H S)$ quantifies the deviation of the humeral head center $(H)$ from the glenoid center $(C)$. The orientation of the scapulohumeral subluxation $(S H S O)$ is represented by the angle of subluxation relative to the anterior-posterior axis $(A P)$. The SHS is based on the scapular axis, whereas the glenohumeral subluxation $(G H S)$ is based on the glenoid centerline. The 3D glenoid version $(G V)$ is the angle between the glenoid centerline and the scapular axis.

within 6 sectors of $60^{\circ}$ each. The 6 sectors were posterior, posteriorsuperior, posterior-inferior, anterior, anterior-superior, and anteriorinferior. We also calculated the (linear) correlation between glenoid version and scapulohumeral subluxation and between glenoid version and glenohumeral subluxation. We correlated the amplitude of the subluxation with the amplitude of the glenoid version and the orientation of the subluxation with the orientation of the glenoid version. The orientation correlations were performed only with subluxed shoulders (subluxation above 5\%) to avoid orientation singularity when subluxation is close to zero. We evaluated the coefficient of determination $\left(R^{2}\right)$ and the $P$ value associated with the null hypothesis such that the slope of the correlation was zero.

For the sake of validation of the 3D method, we compared it with the 2D method for the glenohumeral subluxation, the scapulohumeral subluxation, and the glenoid version. In 2D, the frequency of anterior and posterior subluxation was evaluated. The same correlations we calculated in 3D were also evaluated in 2D. For the sake of clarity, the 3D method illustrated with a typical case is discussed in the next section.

The intraobserver and interobserver variability of our 3D measurements were analyzed with the interclass correlation coefficient. Three observers each randomly repeated the same measurement protocol on the $3 \mathrm{CT}$ images 3 times (for a total of 9 measurements made by each observer). The statistical analysis was done with the Statistics Toolbox of MATLAB (MathWorks Inc).

\section{Results}

The glenohumeral subluxation measured in 2D was $6 \% \pm 5 \%$ (from $0 \%$ to $22 \%$ ). In $3 \mathrm{D}$, the glenohumeral subluxation was $7 \% \pm 4 \%$ (from $0 \%$ to $20 \%$ ). In both the 2D and 3D measurements, nearly half of the glenohumeral subluxations were posterior (Table I). However, in 3D, a large percentage of the posterior subluxations were either in the posterior-superior or posterior-inferior sector. The 2D and 3D glenohumeral subluxations were not statistically different $(P=.083)$ and were poorly correlated $\left(R^{2}=0.226 ; P<.001\right)$.

The scapulohumeral subluxation was $12 \% \pm 11 \%$ (from $0 \%$ to $48 \%$ ) measured in $2 \mathrm{D}$ and $16 \% \pm 9 \%$ (from $0 \%$ to $44 \%$ ) in $3 \mathrm{D}$. In 2D, more than half of the scapulohumeral subluxations were posterior (Table I), but they were primarily posterior $(38 \%)$ and posterior-superior $(31 \%)$ in $3 \mathrm{D}$. The 2D and 3D scapulohumeral subluxations were statistically different $(P<.001)$ and were moderately correlated $\left(R^{2}=0.486 ; P<.001\right)$.

The glenoid version measured in $2 \mathrm{D}$ was $12^{\circ} \pm 9^{\circ}$ (from $0^{\circ}$ to $45^{\circ}$ ). The $3 \mathrm{D}$ version was $15^{\circ} \pm 9^{\circ}$ (from $0^{\circ}$ to $49^{\circ}$ ). In $2 \mathrm{D}$, the version was mainly posterior $(71 \%)$. However, in $3 \mathrm{D}$, it was primarily posterior $(41 \%)$ and posterior-superior $(30 \%)$. The 2D and 3D glenoid versions were statistically different $(P<.01)$ and were moderately correlated $\left(R^{2}=0.540 ; P<.001\right)$.

In $2 \mathrm{D}$, there was no correlation between the glenohumeral subluxation and the glenoid version $\left(R^{2}=0.029\right.$; $P=.071)$, but there was a correlation between the scapulohumeral subluxation and the glenoid version $\left(R^{2}=0.607 ; P<.01\right)$. In 3D, the glenohumeral subluxation and the glenoid version were not correlated in amplitude 
Table I Percentage of cases with glenohumeral subluxation (GHS), scapulohumeral subluxation (SHS), and glenoid version (GV) in the posterior $(P)$ and anterior $(A)$ sides for the $2 \mathrm{D}$ measurements and in the posterior $(P)$, posterior-superior $(P S)$, posterior-inferior $(P I)$, anterior $(A)$, anterior-superior $(A S)$, and anterior-inferior $(A I)$ sectors for the $3 \mathrm{D}$ measurements. For $2 \mathrm{D}$ and $3 \mathrm{D}$ measurement, cases are considered centered $(C)$ when the subluxation is not $>5 \%$ or when the version is not $>5^{\circ}$.

\begin{tabular}{|c|c|c|c|c|c|c|c|}
\hline \multicolumn{4}{|l|}{$2 \mathrm{D}$} & \multicolumn{4}{|l|}{$3 D$} \\
\hline Side & GHS & SHS & GV & GHS & SHS & GV & Sector \\
\hline \multirow[t]{3}{*}{$P$} & $46 \%$ & $56 \%$ & $71 \%$ & $18 \%$ & $38 \%$ & $41 \%$ & $P$ \\
\hline & & & & $13 \%$ & $31 \%$ & $30 \%$ & PS \\
\hline & & & & $4 \%$ & $7 \%$ & $10 \%$ & PI \\
\hline \multirow[t]{3}{*}{ A } & $25 \%$ & $13 \%$ & $6 \%$ & $7 \%$ & $4 \%$ & $4 \%$ & A \\
\hline & & & & $11 \%$ & $6 \%$ & $3 \%$ & AS \\
\hline & & & & $5 \%$ & $3 \%$ & $4 \%$ & AI \\
\hline$C$ & $29 \%$ & $30 \%$ & $23 \%$ & $41 \%$ & $10 \%$ & $9 \%$ & $C$ \\
\hline
\end{tabular}

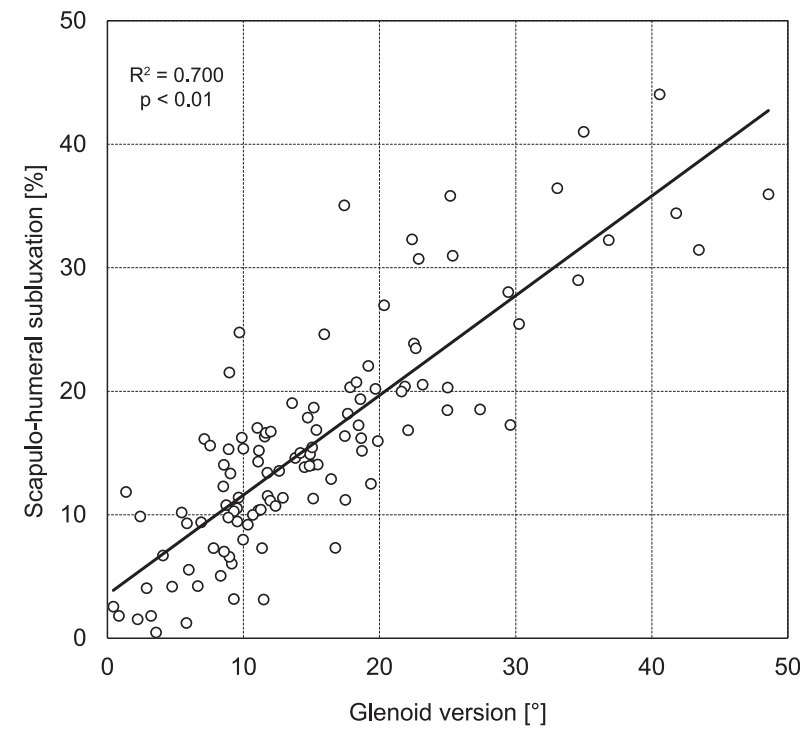

Figure 2 Scapulohumeral subluxation compared with glenoid version in 3D. The amplitude of the scapulohumeral subluxation and the amplitude of the glenoid version were strongly correlated.

$\left(R^{2}=0.020 ; P=.140\right)$, nor were they correlated in orientation $\left(R^{2}<0.001 ; P=.988\right)$. In $3 \mathrm{D}$, the scapulohumeral subluxation and the glenoid version were highly correlated by both their amplitude $\left(R^{2}=0.700\right.$; $P<.01)$ and their orientations $\left(R^{2}=0.864 ; P<.01\right)$. The 2 correlation lines nearly passed through the origin, and their slopes were very close to 1 (Figs. 2 and 3).

The example case (Fig. 4) had no glenohumeral subluxation $(<5 \%)$ when measured in $2 \mathrm{D}$ but a high scapulohumeral subluxation $(32 \%)$ in 3D. The orientation of the scapulohumeral subluxation was in the posterior-superior sector, whereas the CT axial plane used for the 2D measurement was about $60^{\circ}$ different from the orientation of the scapulohumeral subluxation. This is a typical case in which the subluxation is underevaluated by the usual $2 \mathrm{D}$ glenohumeral measure. The scapulohumeral subluxation measured in $2 \mathrm{D}$ was $7 \%$ posterior, and the version measured in $2 \mathrm{D}$ was $15^{\circ}$ posterior. However, the version

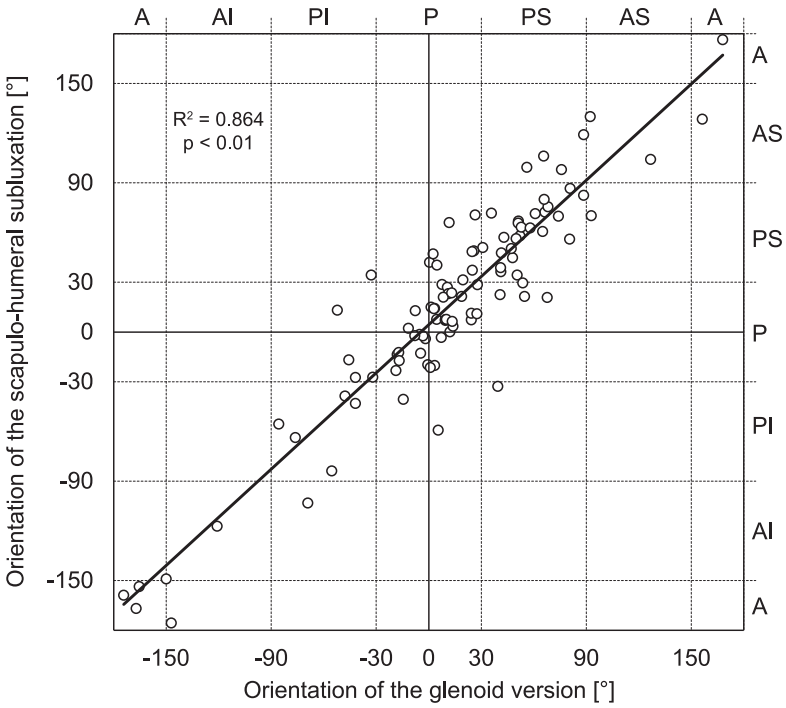

Figure 3 Orientation of the scapulohumeral subluxation compared with the orientation of the glenoid version in 3D in the 6 sectors: posterior $(P)$, posterior-superior $(P S)$, posterior-inferior $(P I)$, anterior $(A)$, anterior-superior $(A S)$, and anterior-inferior $(A I)$. The orientation of the scapulohumeral subluxation and the orientation of the glenoid version were strongly correlated. The orientation of version and the orientation of the subluxation covered all sectors of the glenoid, but they were mainly in the posterior and posterior-superior sectors.

measured in $3 \mathrm{D}$ was $22^{\circ}$ and was oriented in the posteriorsuperior sector $\left(50^{\circ}\right)$. The reliability of the 3D measurement was high (Table II).

\section{Discussion}

The humeral head subluxation and the glenoid version are important parameters to take into account in performing a TSA. They are currently measured on one of the images of the CT sequence that approximately passes through the middle of the glenoid surface. These CT images have a variable orientation relative to the scapula, which can cause 

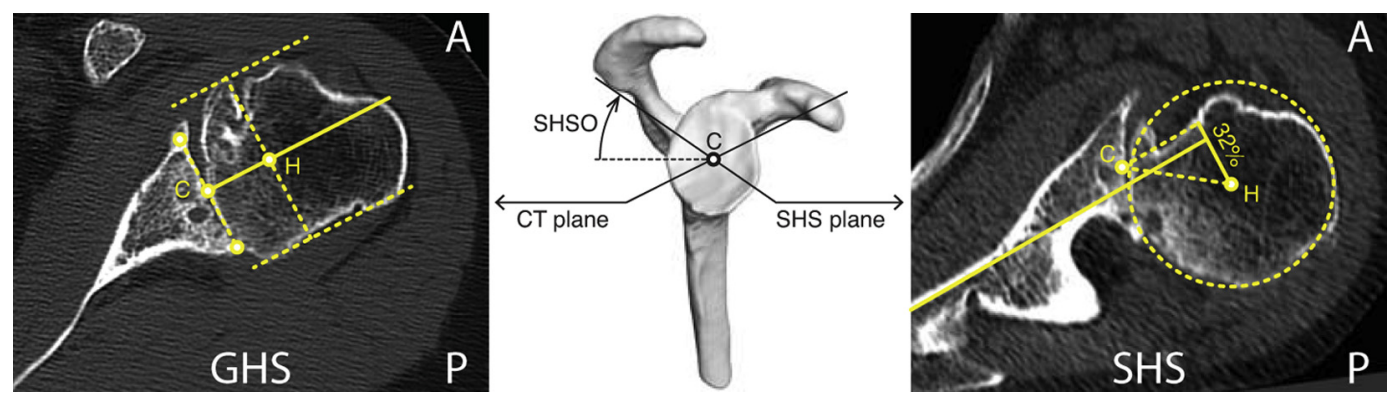

Figure 4 Typical case comparing the usual glenohumeral subluxation $(G H S)$ measured in 2D (left) with the scapulohumeral subluxation (SHS) measured in 3D (right) and their respective measurement plane orientation (center). With the 2D method, the humeral head center $(H)$ is aligned on the glenoid centerline (yellow continuous line). With the 3D method, the humeral head center $(H)$ presents an important posterior subluxation (32\%). The 2D method uses an image from the CT data set that crosses the center of the glenoid $(C)$. The 3D method is based on a 3D geometric analysis, but it is illustrated here in 2D, in the plane of maximal scapulohumeral subluxation (SHS plane), which is characterized by its orientation angle (SHSO). The yellow continuous line represents the scapular axis.

Table II The interobserver and intraobserver interclass correlation coefficient $(I C C)$ for the glenohumeral subluxation (GHS), glenohumeral subluxation orientation (GHSO), scapulohumeral subluxation (SHS), scapulohumeral subluxation orientation (SHSO), glenoid version (GV), and glenoid version orientation (GVO)

\begin{tabular}{lll}
\hline Measure & Interobserver ICC & Intraobserver ICC \\
\hline GHS & 0.97 & 0.88 \\
GHSO & 0.97 & 0.90 \\
SHS & 0.99 & 0.90 \\
SHSO & 0.97 & 0.88 \\
GV & 0.99 & 0.98 \\
GV0 & 0.95 & 0.70 \\
\hline
\end{tabular}

inaccurate measurements. In this study, we extended these 2D measurements to 3D by evaluating not only their amplitude but also their orientation. The objective was to assess the advantage and biomechanical validity of this $3 \mathrm{D}$ measure compared with the usual 2D measure. In 3D, we have observed a strong correlation between scapulohumeral subluxation and the glenoid version, which was not previously reported.

The usual glenohumeral subluxation measurement is performed in 2D. It characterizes the position of the humeral head relative to the glenoid centerline. This measure is quantified as a percentage of the humeral head size. The 3D measures of glenohumeral and scapulohumeral subluxation presented here were built on that same basis as a 3D measure of glenoid version. ${ }^{14}$ They are based on a 3D analysis that generalizes the $2 \mathrm{D}$ measure by quantifying the maximal eccentricity of the humeral head from an ideal centered position and the orientation of this maximal eccentricity. In 3D, we assumed that the humeral head center should be facing the glenoid center along the alignment of the glenoid centerline or along the scapular axis for the glenohumeral or scapulohumeral subluxation, respectively.

The glenohumeral subluxation was lower than the scapulohumeral subluxation, but they could coincide if the glenoid version was zero. The $2 \mathrm{D}$ subluxation was lower than the 3D measure, but they could coincide if the subluxation orientation is the same as the arbitrary CT plane. The subluxation orientation was not strictly in the posterior or anterior sector in more than half of the cases, which emphasizes the importance of taking into account this orientation.

The biomechanical significance of the 3D measurements of subluxation and version relies on the choice of the scapular plane and the scapular axis. The scapular plane is approximately the plane of symmetry of the rotator cuff muscles, and the scapular axis is aligned with the supraspinatus. The validity of the $3 \mathrm{D}$ measurement method proposed here is supported by the strong correlation between scapulohumeral subluxation and glenoid version. Only 5 of the 112 cases presented were outside the $95 \%$ confidence bounds of the version-subluxation correlation. This correlation appeared in the amplitude but also in the orientation of version and subluxation. Every degree of glenoid version was associated with a percentage of subluxation in the same orientation. In our series, the cases outside the correlation bounds were not correlated to any of the measured quantities, nor were they correlated to patient age or sex.

Historically, static humerus subluxation was initially measured in 2D relative to the glenoid surface. Using 70 CT images of osteoarthritic shoulders, Badet et al reported that posterior subluxation was found in $40 \%$ of the cases, but it was not correlated to glenoid retroversion. ${ }^{1}$ Assuming a limit of $5 \%$ of relative glenohumeral subluxation, Walch reported a posterior subluxation of $45 \%$ of 151 primary AO shoulders. ${ }^{17} \mathrm{He}$ reported that subluxation was not correlated with glenoid retroversion or with humeral retroversion. In a following paper, the rate of subluxation (type B in Walch classification) was $32 \%$ in a series of 113 primary osteoarthritic shoulders. ${ }^{16}$ Walch suggested that there was a correlation between subluxation and version but that it was not statistically significant. The 2D measure of glenohumeral subluxation 
proposed by Walch was later adapted to use the scapula as a reference instead of the glenoid. ${ }^{10}$ In the later article, a scapular axis reference line was defined in the first CT image under the coracoid process with the medial border of the scapula and the glenoid center. This scapular axis reference line is also referred to as the transverse axis or the Friedman line. ${ }^{3}$ The scapular axis defined in the present paper can be seen as a $3 \mathrm{D}$ extension of this $2 \mathrm{D}$ scapular axis reference line.

With the 2D definition of the scapular axis, Kidder et al reported that scapulohumeral subluxation was statistically higher than glenohumeral subluxation. ${ }^{10}$ In their study, the scapulohumeral subluxation varied from $37 \%$ to $119 \%$, whereas the glenohumeral subluxation varied from $29 \%$ to $92 \%$. Scapulohumeral subluxation was also more frequent than glenohumeral subluxation, with $100 \%$ of cases exhibiting scapulohumeral subluxation and $58 \%$ exhibiting glenohumeral subluxation. Kidder et al did not report any correlation between scapulohumeral subluxation and version. ${ }^{10}$ Using the same measurement methodology but a subluxation limit of $15 \%$, Gerber et al reported a posterior subluxation in $27 \%$ of 124 osteoarthritic shoulders but no correlation between preoperative humerus subluxation and glenoid version. ${ }^{4}$ In another series of 121 shoulders, Hoenecke et al measured glenohumeral subluxation and glenoid version. Glenohumeral subluxation was measured according to the method of Badet, and the version was measured by 2 different methods. ${ }^{7}$ They concluded that glenoid version did not correlate with humeral subluxation but reported a higher mean subluxation for biconcave glenoids. Overall, our 2D measurements of glenohumeral subluxation, scapulohumeral subluxation, and glenoid version were consistent with the values reported in the literature. ${ }^{8}$

The strength of this study is to extend a well-accepted 2D measurement method to 3D. The 3D method is based on a vector analysis but can also be understood as a $2 \mathrm{D}$ measurement performed in the plane of maximal subluxation instead of the arbitrary plane of the CT. This plane of maximal subluxation passes through the humeral head center and is aligned with the orientation of subluxation. This theoretically guarantees the maximum value of subluxation. As it is defined here, the scapular axis can be related to the biomechanical axis of the rotator cuff muscles, which are critical for the stability of the glenohumeral joint. In addition, the $3 \mathrm{D}$ method can be used with a regular clinical CT study, which usually does not include the entire scapula. For that purpose, we have defined bone landmarks that are not located in typical erosion zones. ${ }^{14}$ Thus we can assume that the 3D measure would not be affected by osteoarthritic changes. The methodology presented here still requires some manual operations. However, for a practical clinical application, the method will be fully automated.

\section{Conclusion}

This work addresses the problem of measuring shoulder subluxation in osteoarthritic glenohumeral joints. Glenoid version and scapulohumeral subluxation are important parameters to take into account in performing a TSA. We have showed that 3D measurement of scapulohumeral subluxation should be preferred to the usual 2D measurement of glenohumeral subluxation. Therefore, we recommend $3 \mathrm{D}$ measurements of the glenoid version and the scapulohumeral subluxation to detect them when they are out of the CT plane, even if the best way to correct them is not yet fully known. However, the strong correlation reported between humerus subluxation and glenoid version does not explain the cause of the subluxation. One could assume that muscle misbalance could also play a role in causing shoulder subluxation, which has the secondary effect of inducing glenoid erosion. This hypothesis still needs to be confirmed.

\section{Acknowledgment}

This project was partly funded by the Inter-Institutional Center for Translational Biomechanics (EPFL-CHUVDAL) and by Tornier (Tornier, Inc, Edina, MN, USA). The authors thank Caroline Sieger Fernandes for language editing and editorial assistance.

\section{Disclaimer}

The authors, their immediate families, and any research foundation with which they are affiliated did not receive any financial payments or other benefits from any commercial entity related to the subject of this article.

\section{References}

1. Badet R, Boileau P, Noel E, Walch G. Arthrography and computed arthrotomography study of seventy patients with primary glenohumeral osteoarthritis. Rev Rhum Engl Ed 1995;62:555-62.

2. Collins D, Tencer A, Sidles J, Matsen F 3rd. Edge displacement and deformation of glenoid components in response to eccentric loading. The effect of preparation of the glenoid bone. J Bone Joint Surg Am 1992; 74:501-7

3. Friedman RJ, Hawthorne KB, Genez BM. The use of computerized tomography in the measurement of glenoid version. J Bone Joint Surg Am 1992;74:1032-7. 
4. Gerber C, Costouros JG, Sukthankar A, Fucentese SF. Static posterior humeral head subluxation and total shoulder arthroplasty. J Shoulder Elbow Surg 2009;18:505-10. http://dx.doi.org/10.1016/j.jse.2009.03.003

5. Gerber C, Nyffeler RW. Classification of glenohumeral joint instability. Clin Orthop Relat Res 2002:65-76.

6. Habermeyer P, Magosch P, Lichtenberg S. Recentering the humeral head for glenoid deficiency in total shoulder arthroplasty. Clin Orthop Relat Res 2007;457:124-32. http://dx.doi.org/10.1097/BLO. 0b013e31802ff03c

7. Hoenecke HR Jr, Tibor LM, D'Lima DD. Glenoid morphology rather than version predicts humeral subluxation: a different perspective on the glenoid in total shoulder arthroplasty. J Shoulder Elbow Surg 2012; 21:1136-41. http://dx.doi.org/10.1016/j.jse.2011.08.044

8. Hsu JE, Ricchetti ET, Huffman GR, Iannotti JP, Glaser DL. Addressing glenoid bone deficiency and asymmetric posterior erosion in shoulder arthroplasty. J Shoulder Elbow Surg 2013;22:1298-308. http://dx.doi.org/10.1016/j.jse.2013.04.014

9. Iannotti JP, Norris TR. Influence of preoperative factors on outcome of shoulder arthroplasty for glenohumeral osteoarthritis. J Bone Joint Surg Am 2003;85:251-8.

10. Kidder JF, Rouleau DM, Pons-Villanueva J, Dynamidis S, Defranco MJ, Walch G. Humeral head posterior subluxation on CT scan: validation and comparison of 2 methods of measurement. Tech Shoulder Elbow Surg 2010;11:72-6. http://dx.doi.org/10.1097/BTE. Ob013e3181e5d742
11. Mizuno N, Denard PJ, Raiss P, Walch G. Reverse total shoulder arthroplasty for primary glenohumeral osteoarthritis in patients with a biconcave glenoid. J Bone Joint Surg Am 2013;95:1297-304. http://dx. doi.org/10.2106/JBJS.L.00820

12. Papilion JA, Shall LM. Fluoroscopic evaluation for subtle shoulder instability. Am J Sports Med 1992;20:548-52.

13. Sabesan V, Callanan M, Ho J, Iannotti JP. Clinical and radiographic outcomes of total shoulder arthroplasty with bone graft for osteoarthritis with severe glenoid bone loss. J Bone Joint Surg Am 2013;95: 1290-6. http://dx.doi.org/10.2106/JBJS.L.00097

14. Terrier A, Ston J, Larrea X, Farron A. Measurements of threedimensional glenoid erosion when planning the prosthetic replacement of osteoarthritic shoulders. Bone Joint J 2014;96-B:513-8. http:// dx.doi.org/10.1302/0301-620X.96B4.32641

15. Walch G, Ascani C, Boulahia A, Nove-Josserand L, Edwards TB. Static posterior subluxation of the humeral head: an unrecognized entity responsible for glenohumeral osteoarthritis in the young adult. J Shoulder Elbow Surg 2002;11:309-14. http://dx.doi.org/10.1067/mse. 2002.124547

16. Walch G, Badet R, Boulahia A, Khoury A. Morphologic study of the glenoid in primary glenohumeral osteoarthritis. J Arthroplasty 1999; 14:756-60.

17. Walch G, Boulahia A, Boileau P, Kempf JF. Primary glenohumeral osteoarthritis: clinical and radiographic classification. The Aequalis Group. Acta Orthop Belg 1998;64(Suppl 2):46-52. 